\title{
Erratum to: Cytokeratin7 and cytokeratin 19 expression in high grade cervical intraepithelial neoplasm and squamous cell carcinoma and their possible association in cervical carcinogenesis
}

Hojung Lee ${ }^{1}$, Hyekyung Lee ${ }^{2}$ and Yong Kyun $\mathrm{Cho}^{3^{*}}$

\section{Erratum}

The original version of this article [1] unfortunately contained incorrect figure files. Figures 4 and 7 in main body which have been replaced with new version of these figures. The corrected Figures include 3 added HE $(\mathrm{a}, \mathrm{b}, \mathrm{c})$ and total $15(\mathrm{a}-\mathrm{o})$ figures, each.

The new version of these files are included below.

Furthermore, Additional File 1 has been updated to remove Fig. 3 and 6 which have already been included in the main body of the article. The original version of this article has been updated to reflect the above changes.

\section{Additional file}

Additional file 1: Figure S1. Low power view (x40) of HE staining (a) and CK7 (b) CK19 (c) p16 (d) and HR HPV (e) expression pattern of SCC\#19 (PPTX $3720 \mathrm{~kb})$

Author details

${ }^{1}$ Department of Pathology, Nowon Eulji Medical Center, Eulji University, 280-1 Hagye 1-dong, Nowon-gu, Seoul 139-711, Korea. ${ }^{2}$ Department of Pathology, Daejeon Eulji Medical Center, Eulji University, 95 Dunsanseo-ro, Seo-gu, Daejeon 35233, Korea. ${ }^{3}$ Department of Internal Medicine, Division of Infectious Diseases, Gachon University Gil Medical Center, 1198 Guwol-dong, Namdong-gu, Incheon 405-760, Korea.

Published online: 22 May 2017

\section{Reference}

1. Lee et al. Diagnostic Pathology (2017) 12:18. Cytokeratin7 and cytokeratin19 expression in high grade cervical intraepithelial neoplasm and squamous cell carcinoma and their possible association in cervical carcinogenesis. doi:10.1186/s13000-017-0609-4

\footnotetext{
* Correspondence: karmacho@gmail.com

${ }^{3}$ Department of Internal Medicine, Division of Infectious Diseases, Gachon University Gil Medical Center, 1198 Guwol-dong, Namdong-gu, Incheon 405-760, Korea

Full list of author information is available at the end of the article
} 


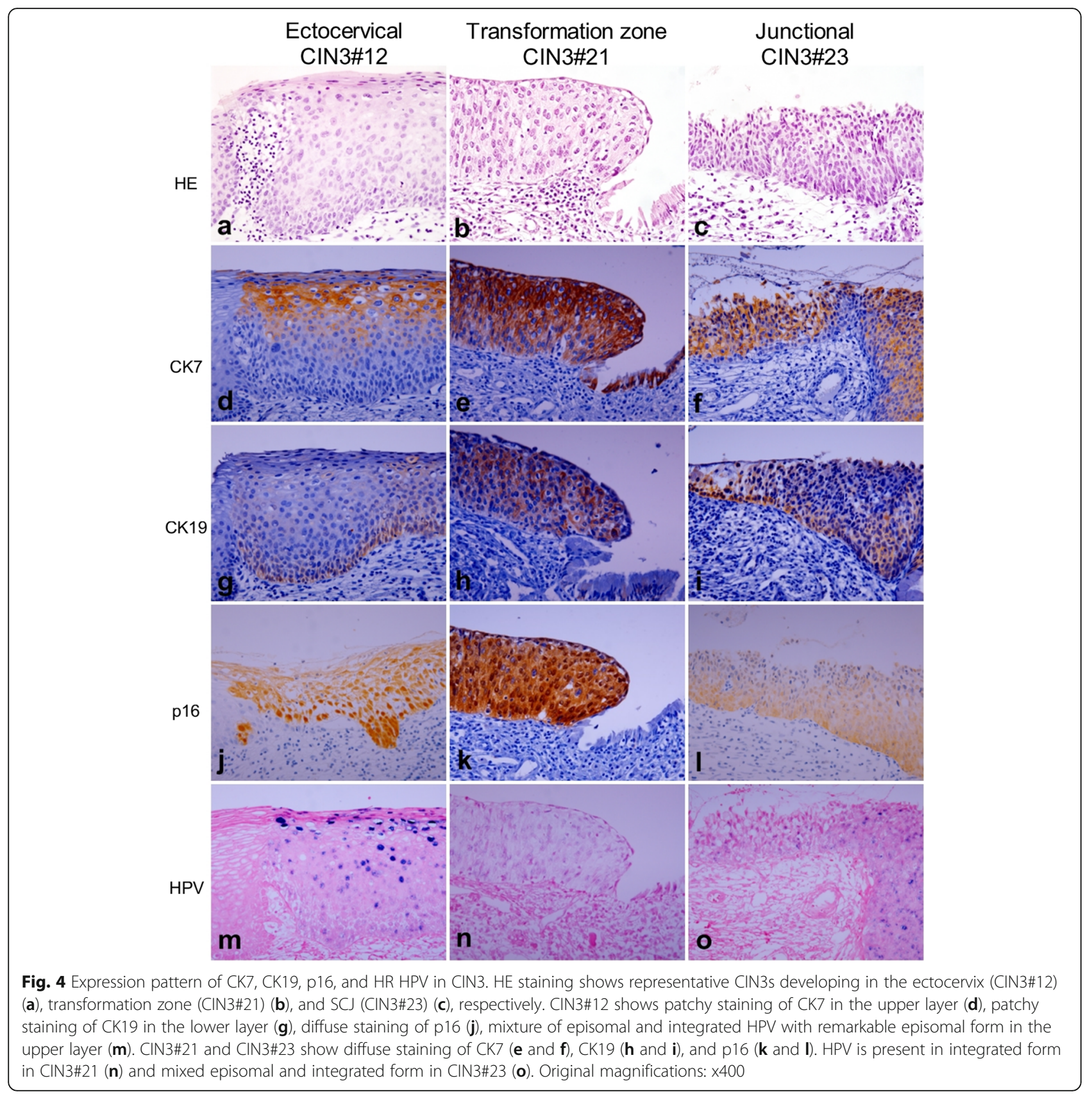




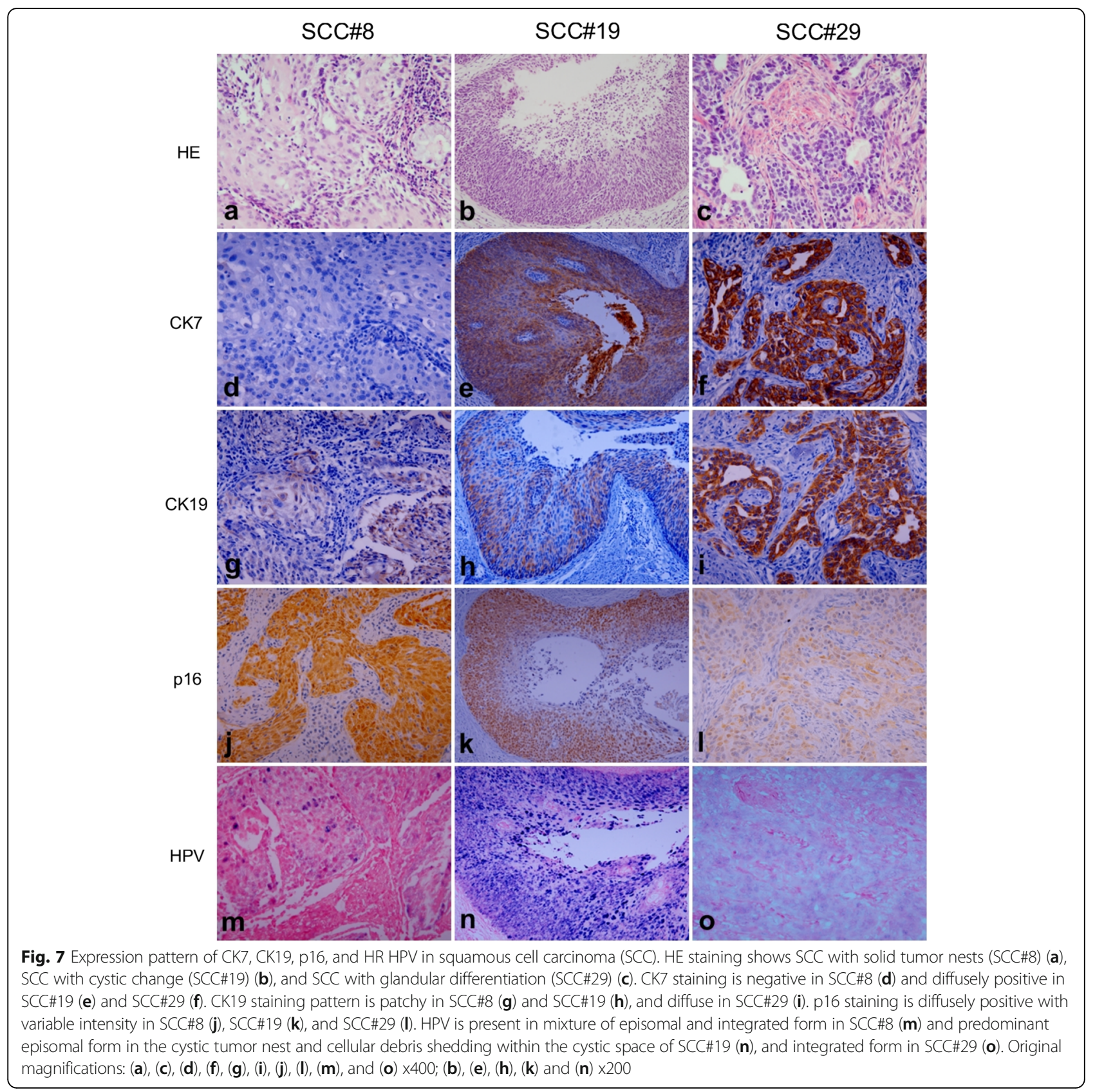

